\title{
Development of Compact High Efficiency Microstructured Semiconductor Neutron Detectors
}

\author{
D.S. McGregor*a, S.L. Bellinger ${ }^{\mathrm{a}, \mathrm{c}}$, R.G. Fronk ${ }^{\mathrm{a}}$, L. Henson ${ }^{\mathrm{c}}$, D. Huddleston ${ }^{\mathrm{b}}, \mathrm{T}$. \\ Ochs $^{\mathrm{a}}$, J.K. Shultis ${ }^{\mathrm{a}}$, T.J. Sobering ${ }^{\mathrm{b}}$, R.D. Taylor ${ }^{\mathrm{b}}$ \\ ${ }^{a}$ SMART Laboratory, Department of Mechanical and Nuclear Engineering, Kansas State \\ University, Manhattan, KS 66506, USA \\ ${ }^{b}$ Electronics Design Laboratory, Kansas State University, Manhattan, KS 66506, USA \\ ${ }^{c}$ Radiation Detection Technologies, Manhattan, KS 66503, USA
}

\begin{abstract}
Semiconductor diode detectors coated with neutron reactive materials are generally fashioned as planar diodes coated with ${ }^{10} \mathrm{~B},{ }^{6} \mathrm{LiF}$, or $\mathrm{Gd}$. Planar detectors coated with ${ }^{10} \mathrm{~B}$ or ${ }^{6} \mathrm{LiF}$ are limited to less than $5 \%$ intrinsic thermal neutron detection efficiency. Detectors coated with Gd can achieve higher efficiencies, but the low-energy signatures are problematic in the presence of background radiations. Microstructured semiconductor neutron detectors (MSND) can now achieve a tenfold increase in neutron detection efficiency over the planar diode designs. These semiconductor neutron detectors are fashioned with a matrix of microstructured patterns etched deeply into the semiconductor substrate and, subsequently, backfilled with neutron reactive materials. Intrinsic thermal-neutron detection efficiencies exceeding $35 \%$ have been achieved with devices no thicker than $1 \mathrm{~mm}$ while operating on less than 5 volts, now allowing for instrumentation to be realized with similar performance as ${ }^{3} \mathrm{He}$ gas-filled detectors.
\end{abstract}

\footnotetext{
${ }^{*}$ Corresponding author

Email address: mcgregor@ksu.edu (D.S. McGregor*)
}

Preprint submitted to Radiation Physics and Chemistry

May 7, 2015 
Key words:

semiconductor neutron detectors, microstructured detectors, MSND

\section{Introduction}

Coated semiconductor detectors have been studied as neutron detectors for over fifty years, dating back to work performed by Babcock et al. [1] (see also [2, 3] and references therein). Neutrons interacting in the reactive coating cause the spontaneous ejection of energetic reaction products. Should one of these reaction products enter the adjacent semiconductor diode, it excites electron-hole pairs whose charge can be measured through a tiny current or through a shaped voltage pulse. The advantages of coated diodes as neutron detectors include compact size, low power requirements, low cost VLSI mass production methodology, and ruggedness. The three most often investigated neutron reactive materials for such a device are ${ }^{10} \mathrm{~B},{ }^{6} \mathrm{LiF}$, or natural $\mathrm{Gd}$. Yet, because the basic planar thin-film diode coated with either ${ }^{10} \mathrm{~B}$ or ${ }^{6} \mathrm{LiF}$ are limited to a practical maximum intrinsic thermal-neutron detection efficiency $\varepsilon_{t n}$ of approximately $4.5 \%$ [4], they have not been widely used as neutron radiation detectors ${ }^{1}$.

Muminov and Tsvang suggested the incorporation of channels in a semiconductor substrate backfilled with a neutron reactive material might increase the neutron detection efficiency of a semiconductor based diode [5]. This idea was later resurrected by Schelten $[6,7]$, yet neither group built or reported working devices. Muminov [5] suggested that the extended surface area of the shallow

\footnotetext{
${ }^{1}$ Detectors coated with pure ${ }^{6} \mathrm{Li}$ metal can achieve $\varepsilon_{t n}>11 \%$ [4], yet the reactivity of Li metal complicates fabrication. Detectors coated with Gd foil can achieve $\varepsilon_{t n}>22 \%$, but only with the lower level discriminator (LLD) set at zero. With the LLD adjusted to a realistic level, such as 80 $\mathrm{keV}$ equivalent, the efficiency drops below $4 \%$ (see brief discussion in [3]).
} 
channels would increase the overall detection efficiency, a suggestion that is only partially true. As pointed out elsewhere [8-10], it is actually the added effects of the extended surface area and the increased probability that reaction products can enter the semiconductor material, due to additional geometric effects, that truly increases the efficiency.

For reasons explained elsewhere $[3,10],{ }^{6} \mathrm{LiF}$ was determined to be the best choice of material, when compared to $\mathrm{Gd}$ and ${ }^{10} \mathrm{~B}$, for this class of radiation detector. The ${ }^{6} \mathrm{Li}(\mathrm{n}, \mathrm{t})^{4} \mathrm{He}$ neutron reaction yields,

$$
{ }_{0}^{1} \mathrm{n}+{ }_{3}^{6} \mathrm{Li} \longrightarrow{ }_{1}^{3} \mathrm{H}(2.7276 \mathrm{MeV})+{ }_{2}^{4} \mathrm{He}(2.0553 \mathrm{MeV})
$$

\footnotetext{
${ }^{2} \mathrm{The}{ }^{6} \mathrm{Li}$ atomic density in ${ }^{6} \mathrm{LiF}$ is actually higher $\left(6.118 \times 10^{22} \mathrm{~cm}^{-3}\right)$ than in pure ${ }^{6} \mathrm{Li}$ metal $\left(4.634 \times 10^{22} \mathrm{~cm}^{-3}\right)$.
} 


\section{MSND Design}

The basic MSND design is shown in Fig. (1), where a few salient features are depicted. The MSND has micromachined features extending into a semiconductor surface. In or about these microstructures is a rectifying contact $[15,16]$. Some of the first generation devices had simple Schottky barrier rectifying contacts; however, almost all recent generation devices have p-type contacts diffused into high-resistivity $10 \mathrm{k} \Omega$-cm n-type Si. Inside the microstructured features is a neutron reactive material. First generation devices were backfilled with ${ }^{10} \mathrm{~B}$ material $[13,14]$; however, almost all recent generation devices are backfilled with ${ }^{6} \mathrm{LiF}$. Finally an ohmic contact is formed on the backplane of the device, depicted as an n-type contact in Fig. (1). In cases where a series of detectors are arranged in an array upon a single wafer, an insulating $\mathrm{SiO}_{2}$ layer is grown between the devices. The generalized MSND fabrication process can be found in the literature $[3,17]$.

Neutrons interacting in the ${ }^{6} \mathrm{LiF}$ material produce energetic reaction products that have a high probability of intersecting the semiconductor material. If the energy deposited within the semiconductor is greater than the threshold established by the lower level discriminator (LLD), the reaction products absorbed in the semiconductor will register a 'count'. The LLD, etched-pattern design, trench width, trench depth, and semiconductor fin width determine the limiting neutron detection efficiency.

\subsection{Expected Performance}

MSNDs were designed and developed with MCNP6, wherein trenches were modeled as $400 \mu \mathrm{m}$ deep in a silicon substrate and backfilled with ${ }^{6} \mathrm{LiF}$. A small- 
diameter, parallel beam of $0.0254 \mathrm{eV}$ neutrons were modeled as irradiating the top side of the device. Neutron absorptions occurring within the ${ }^{6} \mathrm{LiF}$ material generated the appropriate reaction products [4], producing the pulse-height spectra shown in Fig. (2).

Each spectrum shown in Fig. (2), from A to F, is the result of the monte-carlo simulation for the geometries listed in Table 1. All calculations were performed for a $40 \mu \mathrm{m}$ pitch with varying trench and fin widths. The density of $95 \%$ enriched ${ }^{6} \mathrm{LiF}$ is $2.54 \mathrm{~g} \mathrm{~cm}^{-3}$ was used in the calculations [4], thereby, providing an upper limit to the expected efficiency. In each case a discernable valley appears between the lowest energy region and the most salient spectral feature, thereby, establishing a convenient region to set the LLD (where few counts are lost). From Table 1 , the valley is narrowest for thin semiconductor fin widths, but the efficiency is highest. The efficiency is highest because of the increased amount of ${ }^{6} \mathrm{LiF}$ neutron absorber (case A). However, the spectrum is shifted to lower channels (energies) because there is less semiconductor material to absorb the reaction product energies, thereby, producing a narrow valley. For wider fins and narrower trenches, the amount of neutron absorber is reduced, but the energy absorbed in the semiconductor is increased; hence, the discriminator valley is wider, but consequently $\varepsilon_{t n}$ is lower. Note that the natural gamma-ray discrimination is best for case $\mathrm{A}$, in which the narrow semiconductor fin is limited in the amount of energy that can be absorbed by a Compton electron or photoelectron. Hence, the LLD threshold required to establish a high neutron: $\gamma$-ray detection ratio is much less for case $\mathrm{A}$ than case F. Regardless, the LLD can be set twice as high for case F than case A without significant loss in $\varepsilon_{t n}$.

The intrinsic efficiency is also a function of neutron trajectory. With normal 
incident radiation, neutrons can stream through the $\mathrm{Si}$ fin regions without intersecting the ${ }^{6} \mathrm{LiF}$ material. If the MSND is rotated such that neutrons intersect at angle $\theta$ from normal, there is a reduced probability that streaming can occur. In other words, as $\theta$ increases, neutrons that are not absorbed in the first ${ }^{6} \mathrm{LiF}$-filled trench they intersect can be absorbed in adjacent (or subsequent) trenches. The intrinsic thermal neutron detection efficiency (see [18]) dependence is shown in Fig. (3), indicating an expected increase in $\varepsilon_{t n}$ with $\theta$. However, as the neutron trajectory angle $\theta$ is increased, the projected area $(A)$ of the detector decreases as $A \cos \theta[4]$, which consequently decreases the interaction rate and observed count rate. The overall expected count rate compared to the normal condition $\left(\theta=0^{\circ}\right)$, is depicted in Fig. (3), showing a moderately flat response between $0^{\circ}$ to $60^{\circ}$.

\section{Instrumentation}

Compact modular units based on the MSND technology have been developed. The modular unit, and some instruments based on the unit, are briefly described here.

\subsection{Modular Unit}

A modular MSND package with a $4 \mathrm{~cm}^{2}$ MSND is commercially available from Radiation Detection Technologies Inc., shown in Fig. (4), and is marketed as the Domino ${ }^{\mathrm{TM}}$ Neutron Detector [19]. The MSND chip is mounted and wirebonded to a printed circuit board (PCB) that is soldered to low-power readout electronics. The readout electronics were developed by the Electronics Design Laboratory at Kansas State University. The Domino ${ }^{\mathrm{TM}}$ readout electronics have an adjustable LLD, and provides TTL digital output pulses for registered neutron events. The MSNDs installed in the Domino ${ }^{\mathrm{TM}}$ provide a normal incident $\varepsilon_{t n}$ 
ranging between $25 \%$ to $33 \%$, depending on required gamma-ray rejection ratio, ranging from $10^{7}$ to $10^{5}$, respectively. Details describing the Domino ${ }^{\mathrm{TM}}$ and its performance can be found in the literature [20].

In order to form larger-area MSND-based instruments, many Dominoes can be arrayed together to function as a single detector system. Long strings of Dominoes can be formed by connecting them end-to-end. In this fashion, long detectors can be formed, effectively increasing the overall size of the semiconductor-based detector. Capacitance and leakage current ultimately limit the overall size possible for an MSND array; however, array strings up to 1 meter length are possible before increases in capacitance and leakage current, primarily from limitations of the integrated electronics, become problematic. This modular system can be further expanded into a second and third dimension for various radiation detection applications.

\subsection{Briefcase Array}

A portable, high-neutron-sensitivity device can be fashioned by tiling multiple strings of MSNDs side-by-side using a summing mother board, effectively summing their signals together to behave as a single, large-area device. A Briefcase Neutron Detector was developed to showcase the light-weight, rugged design, pictured in Fig. (5). The detector system is capable of deploying 168 individual Dominoes with a $12 \mathrm{~V}, 0.5$ A power supply with a detector active area of 12 in $\times$ 15 in $(30.48 \mathrm{~cm} x 38.1 \mathrm{~cm})$. A 1 in $(2.54 \mathrm{~cm})$ thick $\times 12$ in $(30.48 \mathrm{~cm}) \times 15$ in $(38.1 \mathrm{~cm})$ sheet of high density polyethylene (HDPE) was mounted on the front and the back of the detector array, weighing approximately $21 \mathrm{lbs}$. The count rate reported by each Domino ${ }^{\mathrm{TM}}$ in the detector system is summed together at the motherboard and provided as an output via a $5 \mathrm{~V}$ square-wave TTL pulse that is 
generated with $50 \Omega$ impedance. The Briefcase Neutron Detector was tested in a $2.74 \mathrm{~m} \times 2.44 \mathrm{~m}$ hallway composed of concrete cinder block walls within the Kansas State University nuclear reactor building. The detector was tested with a ${ }^{252} \mathrm{Cf}$ spontaneous fission source, which recorded a net count rate of $0.54 \mathrm{cps} \mathrm{ng}^{-1}$ at a distance of $1 \mathrm{~m}$ from a bare ${ }^{252} \mathrm{C}$ f source (when populated with 84 Dominoes). The general results for the ${ }^{252} \mathrm{Cf}$ tests are summarized in Table 2 . It becomes apparent from the data that that efficiency and count rates do not follow the expected $r^{-2}$ dependence, most likely a consequence of the combined effects of parallax and albedo neutrons.

\subsection{Panel Array}

A large area non-portable Panel Array Neutron Detector, shown in Fig. (6), was also developed. The Panel Array Neutron Detector system had 24 strings with 20 Dominoes per string, totaling 480 Dominoes. While operating the entire detector system with a $12 \mathrm{~V}$ source, the Panel Array power consumption was reduced to approximately $1 \mathrm{~A}$. The system was supported by an aluminum frame and had a $1 \mathrm{~m} \times 1 \mathrm{~m} \times 1.5 \mathrm{in}(3.81 \mathrm{~cm})$ thick HDPE moderator behind the array. The detector system was designed such that the count rate from each string could be determined separate from each other, unlike with the Briefcase Neutron Detector that provided a single integrated output. The count rates for each MSND string are recorded on a USB-connected LabView program. Presently, rudimentary 1-dimensional location information can be determined, but further studies are ongoing. The Panel Array Neutron Detector was tested in a 2.74 m x 2.44 m hallway composed of concrete cinder block walls within the Kansas State University nuclear reactor building. Neutron sensitivity tests were performed at distances of $1 \mathrm{~m}, 2 \mathrm{~m}, 5 \mathrm{~m}$ and $10 \mathrm{~m}$ from a $60 \mathrm{ng}{ }^{252} \mathrm{Cf}$ source. At a distance of 2 meters, 
the panel array yielded a net count rate of $1.45 \mathrm{cps} \mathrm{ng}^{-1}$ with a background count rate of $0.893 \mathrm{cps}$. Again it was apparent that the efficiency and count rates did not follow the expected $r^{-2}$ dependence, suspected to be a consequence of parallax and albedo neutrons. The general results for the ${ }^{252} \mathrm{Cf}$ tests are summarized in Table 3.

\section{4. ${ }^{3}$ He Replacement Detector}

A modified Domino ${ }^{\mathrm{TM}}$ detector system was also arrayed into a cylindrical format to test for the possibility of directly replacing aging ${ }^{3} \mathrm{He}$ gas-based neutron detector systems. A 2-in $(5.08 \mathrm{~cm})$ diameter, 6-in $(15.24 \mathrm{~cm})$ long Helium Replacement (HeRep MkII) was built using thirty $4 \mathrm{~cm}^{2}$ active area MSNDs and modified Domino $^{\mathrm{TM}}$ electronics, shown in Fig. (7). The HeRep MkII is powered with a $12 \mathrm{~V}$ power supply and draws $130 \mathrm{~mW}$ under maximum count rate, $30 \mathrm{~mW}$ resting. The specifications for the HeRep MkII are listed in Table 4. The portable detector system is programmed with bias and LLD threshold settings prior to use and can then operate without the use of external electronics, with the exception of a power supply. The count rates reported by each MSND in the detector system are summed at the motherboard, and then a single $5 \mathrm{~V}$ square-wave TTL pulse is generated with $50 \Omega$ impedance. The HeRep MkII was compared directly to a similarly-sized $4 \mathrm{~atm}{ }^{3} \mathrm{He}$ proportional counter using a $60 \mathrm{ng}{ }^{252} \mathrm{Cf}$ source at a distance of one meter. Two tests were performed, where both detectors were placed in identical circumstances. In the first test, the detectors were tested without the aid of a moderator. The ${ }^{3} \mathrm{He}$ proportional counter recorded a net count rate of $3.35 \pm 0.046$ cps while the HeRep MkII recorded a net count rate of $3.19 \pm 0.050$ cps. The detectors were also tested while placed into a 6-in $(15.24 \mathrm{~cm})$ diameter, 9-in $(22.86 \mathrm{~cm})$ tall cylinder of HDPE. The ${ }^{3} \mathrm{He}$ proportional counter and 
the HeRep MkII count rates were $17.13 \pm 0.099$ cps and $17.60 \pm 0.102 \mathrm{cps}$, respectively. The general results for the ${ }^{252} \mathrm{Cf}$ tests are listed in Table 5. The HeRep MkII is capable of containing its own moderator within the detector volume due to the solid-state nature of the constituent devices. More details on these devices can be found in the literature [21].

\section{Conclusions and Future Work}

Microstructured semiconductor neutron detectors (MSND) are now routinely fabricated with $>30 \% \varepsilon_{t n}$. The detectors are mass produced in compact packages as either $1 \mathrm{~cm}^{2}$ or $4 \mathrm{~cm}^{2}$ detectors. Further, these compact packages can be connected and integrated to form large area arrays. Future detectors will be designed as double-sided devices, thereby, practically eliminating neutron streaming problems while doubling $\varepsilon_{t n}$ (see basic design [16]).

\section{Acknowledgement}

This work is supported in part by the Defense Threat Reduction Agency, contract HDTRA1-12-C-0004.

\section{References}

[1] R.V. Babcock, R.E. Davis, S.L. Ruby, K.H. Sun, E.D. Wolley, Nucleonics, 17 (1959) pp. 116-122.

[2] Bibliographical Series No. 18, Neutron Detectors, International Atomic Energy Agency, Vienna, 1966 (references therein). 
205

206

207

208

209

210

211

212

213

214

215

216

217

218

219

220

[3] D.S. McGregor, S.L. Bellinger, J.K. Shultis, J. Crys. Growth, 379 (2013) 99.

[4] D.S. McGregor, M.D. Hammig, Y-H Yang, H.K. Gersch, R.T. Klann, Nucl. Instr. and Meth., A500 (2003) 272.

[5] R.A. Muminov, L.D. Tsvang, Sov. At. Energy, 62 (1987) 255.

[6] J. Schelten, M. Balzhäuser, F. Höngesberg, R. Engels, R. Reinartz, Physica B, 234-236 (1997) 1084.

[7] J. Schelten, R.Reinartz, Neutron Detector, US Patent 58804762, allowed March 9, 1999.

[8] J.K. Shultis and D.S. McGregor, Conf. Rec. IEEE Nucl. Sci. Symp., Rome, Italy, Oct. 18-22, 2004.

[9] J.K. Shultis and D.S. McGregor, IEEE Trans. Nucl. Sci., NS-53 (2006) 1659.

[10] J.K. Shultis, D.S. McGregor, Nucl. Instr. and Meth., A606 (2009) 608.

[11] D.I. Garber, R.R. Kinsey, BNL 325: Neutron Cross Sections, 3rd ed., Vol. 2, Curves, Brookhaven National Laboratory, Upton (1976). 
[12] V. McLane, C.L. Dunford, P.F. Rose, in: Neutron Cross Sections, Vol. 2, Academic Press, San Diego, 1988.

[13] D.S. McGregor, R.T. Klann, H.K. Gersch, E. Ariesanti, J.D. Sanders, and B. Van Der Elzen, Conf. Rec. IEEE Nucl. Sci. Symp., San Diego, California, Nov. 4-9, 2001.

[14] D.S. McGregor, R.T. Klann, H.K. Gersch, E. Ariesanti, J.D. Sanders, and B. Van Der Elzen, IEEE Trans. Nucl. Sci., 49 (2002) 1999.

[15] D.S. McGregor and R.T. Klann, US Patent 6545281; allowed April 8, 2003.

[16] D.S. McGregor, R.T. Klann, US Patent 7164138; allowed January 16, 2007.

[17] S.L. Bellinger, R.G. Fronk, and D.S. McGregor, US Patent 8778715, allowed Jul. 15, 2014.

[18] D.S. McGregor, J.K. Shultis, Nucl. Instrum. and Meth., A632 (2011) 167.

[19] Radiation Detection Technologies, Inc., Domino ${ }^{\mathrm{TM}}$ Neutron Detector D22S20-D0010-V3, http://radectech.com/content/Domino_V3.pdf, accessed June $2,2014$. 
237 [20] S.L. Bellinger, B.W Cooper, R.G. Fronk, T. Ochs, L. Henson, T.J. Sobering, 238 D.S. McGregor, Conf. Proceedings IEEE Nucl. Sci. Symp., Seoul, Korea, $239 \quad$ Oct. 27 - Nov. 2, 2013.

240

241

242
[21] R.G. Fronk, S.L. Bellinger, L. Henson, T. Ochs, T.J. Sobering, D.S. McGregor, Nucl. Instrum. Meth., A779 (2015) 25.

[22] D.S. McGregor, J.K. Shultis, Nucl. Instr. and Meth., A632 (2011) 167. 


\section{Tables}

Table 1. Calculated efficiencies for the spectra of Fig. (2) as a function of fin/trench width and LLD. The total pitch (fin + trench width) was $40 \mu \mathrm{m}$ and total trench depth was 400 $\mu \mathrm{m}$ for all cases.

\begin{tabular}{lllllll}
\hline & $\mathrm{A}$ & $\mathrm{B}$ & $\mathrm{C}$ & $\mathrm{D}$ & $\mathrm{E}$ & $\mathrm{F}$ \\
fin & $10 \mu \mathrm{m}$ & $12 \mu \mathrm{m}$ & $14 \mu \mathrm{m}$ & $16 \mu \mathrm{m}$ & $18 \mu \mathrm{m}$ & $20 \mu \mathrm{m}$ \\
trench & $30 \mu \mathrm{m}$ & $28 \mu \mathrm{m}$ & $26 \mu \mathrm{m}$ & $24 \mu \mathrm{m}$ & $22 \mu \mathrm{m}$ & $20 \mu \mathrm{m}$ \\
\hline$\underline{\mathrm{LLD}}$ & & & & & & \\
0 & $36.3 \%$ & $35.3 \%$ & $34.1 \%$ & $32.6 \%$ & $31.0 \%$ & $29.2 \%$ \\
$300 \mathrm{keV}$ & $34.0 \%$ & $33.3 \%$ & $32.3 \%$ & $31.1 \%$ & $29.7 \%$ & $28.1 \%$ \\
$500 \mathrm{keV}$ & $32.3 \%$ & $31.9 \%$ & $31.1 \%$ & $30.1 \%$ & $28.8 \%$ & $27.4 \%$ \\
\hline
\end{tabular}

Table 2. Detection results for the briefcase array for a $60 \mathrm{ng}{ }^{252} \mathrm{Cf}$ source.

\begin{tabular}{cccc}
\hline Distance & $\begin{array}{c}\text { Count Rate } \\
\text { (cts/s/ng) }\end{array}$ & $\begin{array}{c}\text { Absolute } \\
\text { Efficiency [22] }\end{array}$ & $\begin{array}{c}\text { Intrinsic } \\
\text { Efficiency [22] }\end{array}$ \\
\hline 1 meter & $0.54 \pm 0.02$ & $0.047 \pm 0.00174 \%$ & $5.22 \pm 0.0019 \%$ \\
2 meters & $0.27 \pm 0.01$ & $0.0235 \pm 0.0009 \%$ & $10.1 \pm 0.0038$ \\
5 meters & $0.08 \pm 0.01$ & $0.007 \pm 0.0009 \%$ & $18.85 \pm 0.0038$ \\
\hline
\end{tabular}


Table 3. Detection results for the panel array for a $60 \mathrm{ng}{ }^{252} \mathrm{Cf}$ source.

\begin{tabular}{ccccc}
\hline Distance & Count Rate (cps) & $\begin{array}{c}\text { Count Rate } \\
\text { (cts/s/ng) }\end{array}$ & $\begin{array}{c}\text { Absolute } \\
\text { Efficiency [22] }\end{array}$ & $\begin{array}{c}\text { Intrinsic } \\
\text { Efficiency [22] }\end{array}$ \\
\hline 1 meter & $172.48 \pm 0.76$ & $3.15 \pm 0.014$ & $0.27 \pm 0.0012 \%$ & $4.27 \pm 0.0019 \%$ \\
2 meters & $79.57 \pm 0.52$ & $1.45 \pm 0.009$ & $0.13 \pm 0.0008 \%$ & $6.74 \pm 0.0419$ \\
5 meters & $24.60 \pm 0.21$ & $0.45 \pm 0.004$ & $0.0391 \pm 0.0003 \%$ & $12.23 \pm 0.11$ \\
10 meters & $6.098 \pm 0.066$ & $0.111 \pm 0.001$ & $0.0097 \pm 0.0001 \%$ & $12.16 \pm 0.11$ \\
Background (room) & $0.811 \pm 0.021$ & N/A & N/A & N/A \\
Background (hall) & $0.893 \pm 0.022$ & N/A & N/A & N/A \\
\hline
\end{tabular}

Table 4. HeRep MkII Specifications.

\begin{tabular}{ll}
\hline MSND Area & $4 \mathrm{~cm}^{2}$ \\
\hline Num. of MSNDs & 30 \\
Total Active Area & $120 \mathrm{~cm}^{2}$ \\
Efficiency of each MSND & $20 \%\left(2200 \mathrm{~m} \mathrm{~s}^{-1}\right.$ neutrons) \\
Lower Level Discriminator & $500 \mathrm{keV}$ \\
Total Applied Voltage & $12 \mathrm{volts}$ \\
Power (rest) & $360 \mathrm{~mW}(30 \mathrm{~mA})$ \\
Power (active) & $1.56 \mathrm{~W}(130 \mathrm{~mA})$ \\
\hline
\end{tabular}

Table 5. Comparison responses between a 4 atm ${ }^{3} \mathrm{He}$ detector and the HeRep MkII detector for a $60 \mathrm{ng}{ }^{252} \mathrm{Cf}$ source; source distance $1 \mathrm{~m}$; duration $30 \mathrm{~min}$.

\begin{tabular}{lll}
\hline Device & Count Rate (cps) & Ratio to ${ }^{3}$ He Performance \\
\hline${ }^{3}$ He Tube (bare) & $3.35 \pm 0.046$ & $100 \%$ \\
HeRep MkII (bare) & $3.19 \pm 0.050$ & $95.15 \pm 9.04 \%$ \\
${ }^{3}$ He Tube (HDPE) & $17.13 \pm 0.099$ & $100 \%$ \\
HeRep MkII (HDPE) & $17.60 \pm 0.102$ & $102.74 \pm 2.65 \%$ \\
\hline
\end{tabular}




\section{Figure Captions}

Figure 1. Basic design of a microstructured semiconductor neutron detector (MSND).

Figure 2. Calculated spectral features for various MSNDs, with the pitch held at $40 \mu \mathrm{m}$ and trench depth at $400 \mu \mathrm{m}$, all backfilled with ${ }^{6} \mathrm{LiF}$.

Figure 3. Calculated MSND efficiency as a function of rotational angle from normal. The performance was calculated for MSNDs backfilled with ${ }^{6} \mathrm{LiF}$ with $40 \mu \mathrm{m}$ pitch, $20 \mu \mathrm{m}$ wide trenches and fins, and LLD $=1 \mathrm{MeV}$.

Figure 4. Commercial MSND units. Clockwise from top left (1) sealed Domino $^{\mathrm{TM}}$ MSND, (2) Domino ${ }^{\mathrm{TM}}$ without lid, (3) $2 \mathrm{~cm} \times 2 \mathrm{~cm}$ MSND chip, (4) $1 \mathrm{~cm} \times 1 \mathrm{~cm}$ mounted MSND, (5) $1 \mathrm{~cm} \times 1 \mathrm{~cm}$ MSND chip in a ceramic package.

Fig. 5. The Briefcase Neutron Detector System has Dominoes tiled in two dimensions to form a large-area, portable neutron detector system. The current system is capable of housing 168 Dominoes powered with a $12 \mathrm{~V}, 0.5$ A power supply.

Fig. 6. The MSND neutron detector panel array system while under construction. Twenty-four strings, each comprised of twenty Dominoes, for a total of 480, were arrayed together. The count rate for each string is read out individually and summed via a LabView program. Not pictured is the frame and HDPE moderator used during ${ }^{252} \mathrm{Cf}$ testing.

Fig. 7. The 2nd Generation trefoil MSND-based ${ }^{3}$ He-Replacement (HeRep MkII) neutron detector. The HeRep MkII is shown on the right in each image, 
267 compared to a similarly-sized ${ }^{3} \mathrm{He}$ proportional counter, shown on the left in each 268 image. Installed in the HeRep MkII are thirty $4 \mathrm{~cm}^{2}$ MSNDs, a design developed 269 for replacement of aging ${ }^{3} \mathrm{He}$-based detector systems. 
$270 \quad$ Figures

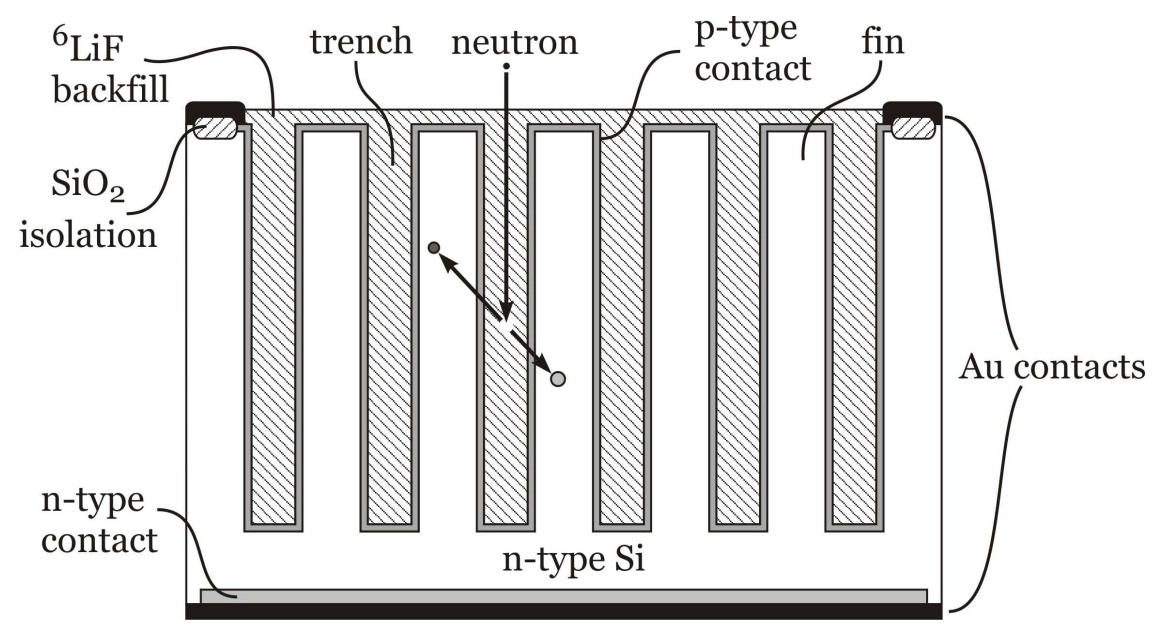

Fig. 1. Basic design of a microstructured semiconductor neutron detector (MSND). 


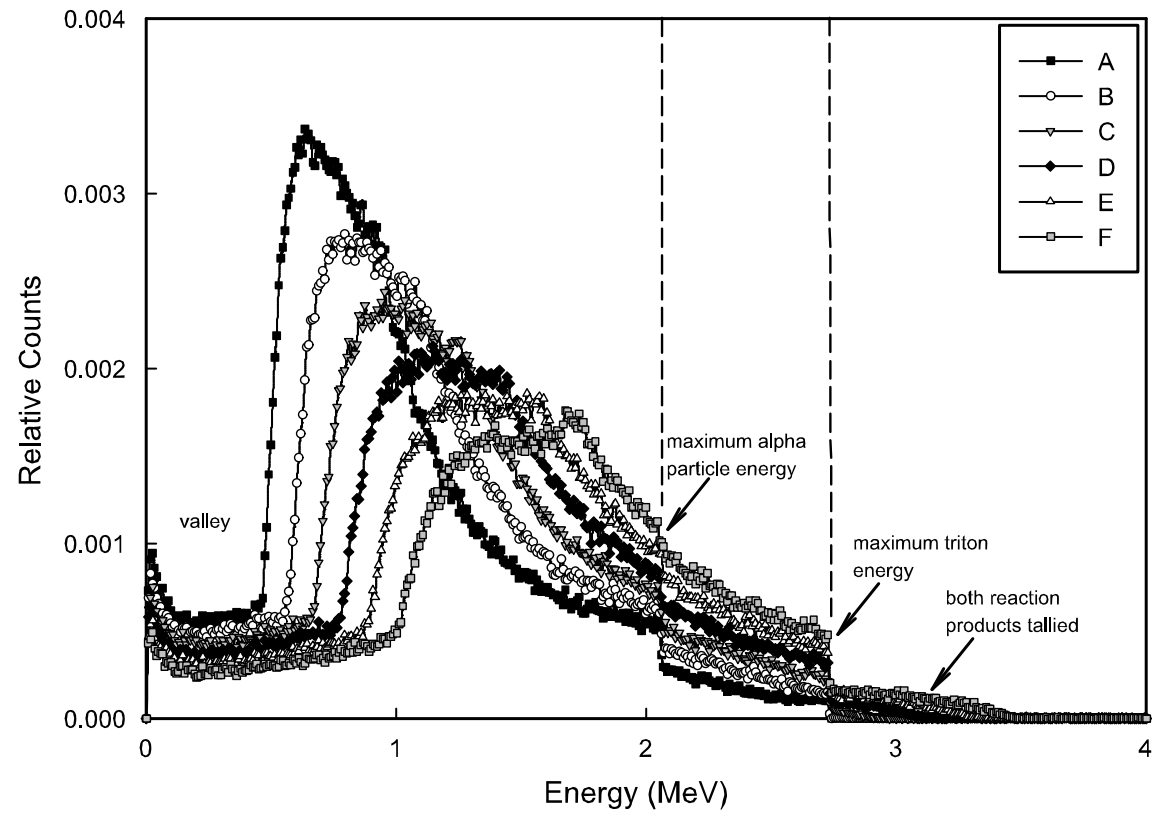

Fig. 2. Calculated spectral features for various MSNDs, with the pitch held at $40 \mu \mathrm{m}$ and trench depth at $400 \mu \mathrm{m}$, all backfilled with ${ }^{6} \mathrm{LiF}$. 


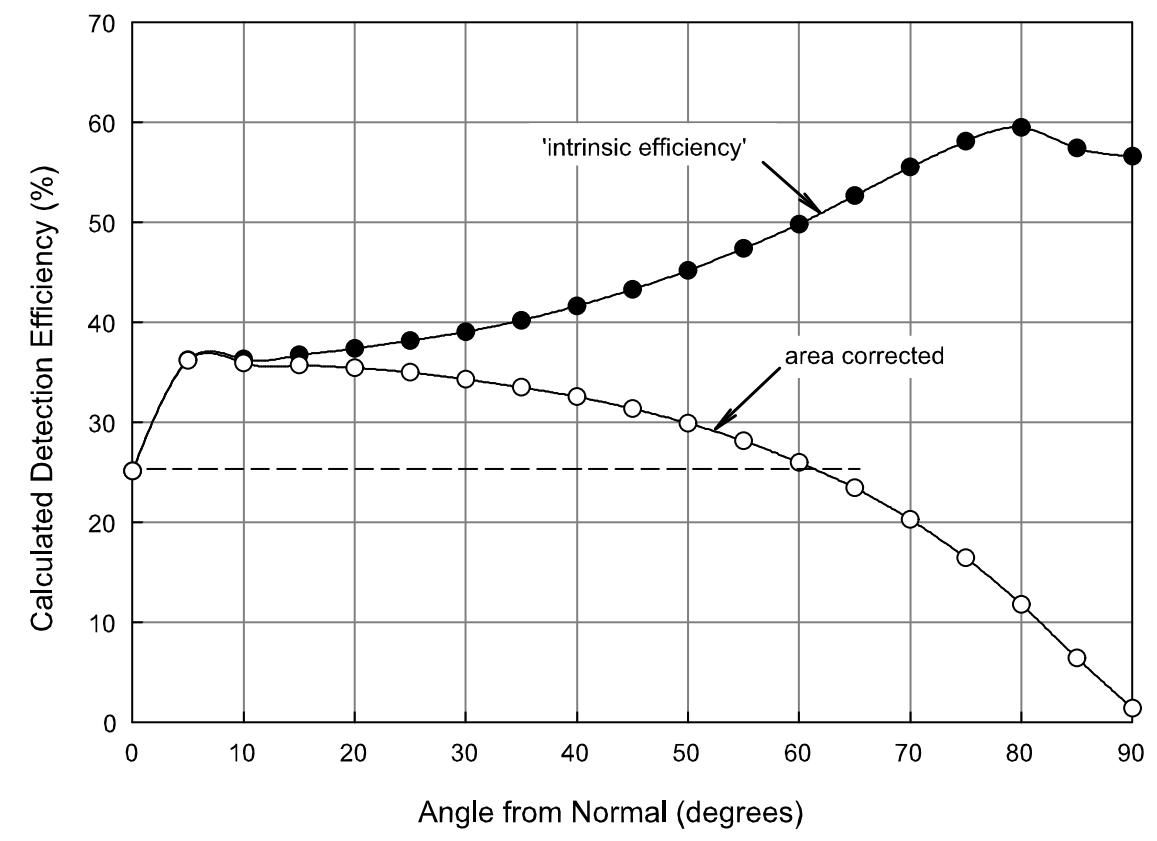

Fig. 3. Calculated MSND efficiency as a function of rotational angle from normal. The performance was calculated for MSNDs backfilled with ${ }^{6} \mathrm{LiF}$ with $40 \mu \mathrm{m}$ pitch, $20 \mu \mathrm{m}$ wide trenches and fins, and LLD $=1 \mathrm{MeV}$.

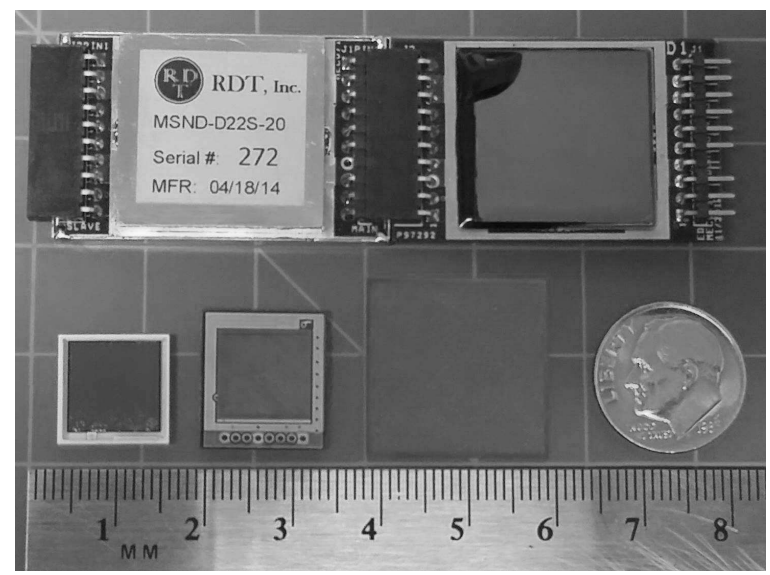

Fig. 4. Commercial MSND units. Clockwise from top left (1) sealed Domino ${ }^{\mathrm{TM}}$ MSND, (2) Domino ${ }^{\mathrm{TM}}$ without lid, (3) $2 \mathrm{~cm} \times 2 \mathrm{~cm}$ MSND chip, (4) $1 \mathrm{~cm} \times 1 \mathrm{~cm}$ mounted MSND, (5) $1 \mathrm{~cm} \times 1 \mathrm{~cm}$ MSND chip in a ceramic package. 
Fig. 5. The Briefcase Neutron Detector System has Dominoes tiled in two dimensions to form a large-area, portable neutron detector system. The current system is capable of housing 168 Dominoes powered with a 12 V, 0.5 A power supply.

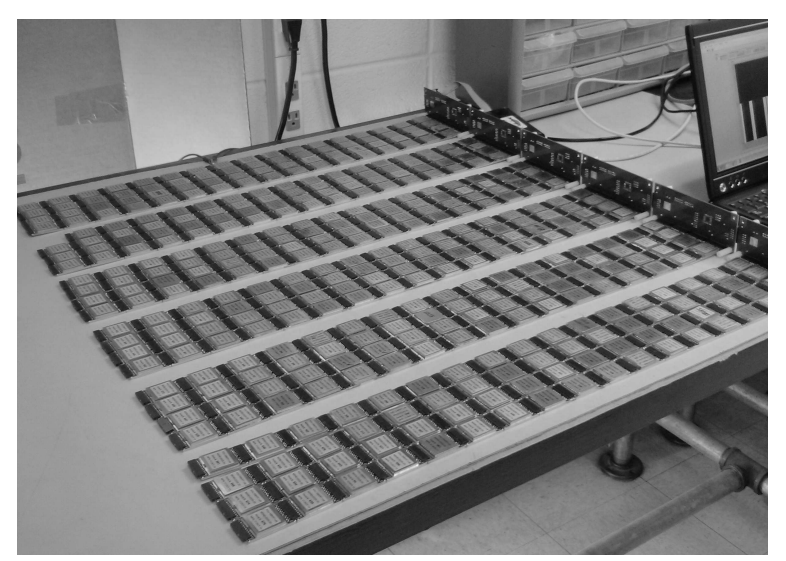

Fig. 6. The MSND neutron detector panel array system while under construction. Twentyfour strings, each comprised of twenty Dominoes, for a total of 480, were arrayed together. The count rate for each string is read out individually and summed via a LabView program. Not pictured is the frame and HDPE moderator used during ${ }^{252} \mathrm{Cf}$ testing. 


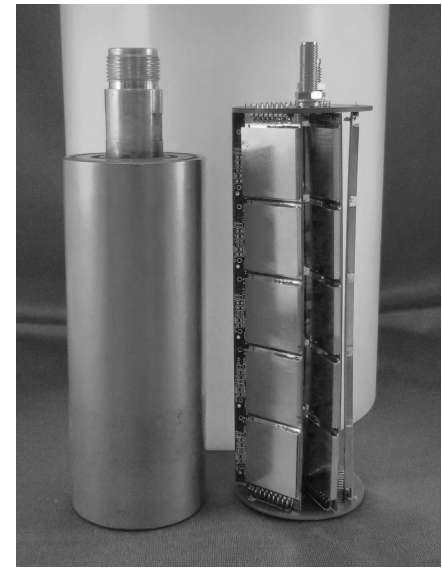

(a)

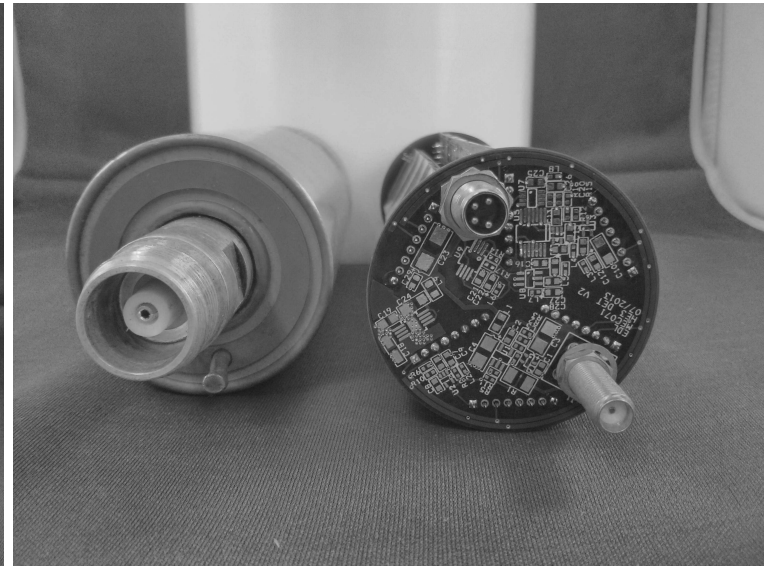

(b)

Fig. 7. The 2nd Generation trefoil MSND-based ${ }^{3} \mathrm{He}-\mathrm{Replacement}$ (HeRep MkII) neutron detector. The HeRep MkII is shown on the right in each image, compared to a similarlysized ${ }^{3} \mathrm{He}$ proportional counter, shown on the left in each image. Installed in the HeRep MkII are thirty $4 \mathrm{~cm}^{2}$ MSNDs, a design developed for replacement of aging ${ }^{3} \mathrm{He}$-based detector systems. 